\title{
First Diagnosis of Inflammatory Bowel Disease in a 91-Year-Old Man
}

\author{
loannis Koutsounas Emmanouil Pyleris \\ Panagiotis Karantanos Charalambos Barbatzas \\ Department of Gastroenterology and Endoscopy Unit, Sismanogleion General \\ Hospital, Marousi, Greece
}

\section{Key Words}

Inflammatory bowel disease - Ulcerative colitis - Crohn's disease - Bloody diarrhea . Elderly

\begin{abstract}
Inflammatory bowel diseases (IBDs) are diseases that occur primarily in adolescence and early adult life. A second peak of IBD incidence occurs at the age of 50-80 years, while reports of first diagnosis after the age of 80 years are extremely rare. It is difficult to establish the true incidence of IBD in older patients due to problems of case definition, population, and particularly because it may be confused with other clinical conditions. A 91-year-old man was admitted to the Emergency Department with progressively worsening abdominal pain and 2-4 episodes of bloody diarrhea daily for the last month. Similar symptoms were not reported by the patient or his family during the past. Complete blood count and biochemical tests were normal, while stool examination showed erythrocytes and white blood cells. Pelvic CT showed inflammatory changes and loss of homogeneity in the perirectal fat together with considerable bowel wall thickening of both the rectum and sigmoid. Colonoscopy revealed edema, hyperemia and spontaneous friability, as well as microulcerations of the rectosigmoid mucosa. Tissue biopsies revealed histopathological lesions compatible with IBD. Finally the patient was treated with metronidazole, ciprofloxacin and mesalazine, with clear clinical improvement during the 5th day of treatment, and was finally discharged with almost normal stools. In conclusion, we report the case of first diagnosis of IBD in a 91-year-old man. The prevalence of IBD in patients aged $>80$ years is difficult to determine. Diagnostic tools are the same as for other age groups, but diagnosis may be difficult because there are a number of clinical conditions that may mimic IBD at this age. The treatment options are those used in younger patients, but special precautions should be taken.
\end{abstract}




\section{Introduction}

Inflammatory bowel diseases (IBDs), including ulcerative colitis (UC) and Crohn's disease (CD), are chronic, progressive and often relapsing inflammatory disorders of the gastrointestinal tract. Their etiology remains not well defined, as genetic, environmental and microbial factors have been reported to play a role in the pathogenesis of IBD. IBD may occur at any time from early childhood to late adulthood, although $>80 \%$ of cases are diagnosed in the second or third decade of life. IBD has a bimodal peak of incidence, with the second peak between the ages of 50 and 80 years. Approximately $15 \%$ of cases manifest after the age of 65 years, with a similar distribution between UC and CD. In the West, the incidence and prevalence of IBDs has increased in the past 50 years, up to $8-14 / 100,000$ and $120-200 / 100,000$ persons, respectively, for UC and 6-15/100,000 and 50-200/100,000 persons, respectively, for $\mathrm{CD}[1]$.

The clinical presentation of UC depends on the extent of the disease process. Patients usually present with diarrhea mixed with blood and mucus, of gradual onset that persists for an extended period. They may also have weight loss and blood on rectal examination. The chronic loss of blood from the gastrointestinal tract leads to increased rates of anemia. The disease may be accompanied by different degrees of abdominal pain, from mild discomfort to painful bowel movements or painful abdominal cramping with bowel movements. Abdominal pain may be the initial symptom of CD. It is often accompanied by diarrhea (bloody or not). The nature of the diarrhea in CD depends on the part of the small intestine or colon involved. Ileitis typically results in large-volume, watery feces. Colitis may result in a smaller volume of feces of higher frequency. Flatulence and bloating may also add to the intestinal discomfort. Systemic symptoms include fever, malabsorption and growth failure. Finally, extraintestinal manifestations include, among others, seronegative spondyloarthropathy, pyoderma gangrenosum, autoimmune hemolytic anemia and neurological complications such as peripheral neuropathy and depression $[2,3]$.

UC is characterized by inflammation and ulcerations in the large bowel mucosa and submucosa, whereas CD is a transmural inflammatory disorder that may involve various sites of the gastrointestinal tract. UC involves the rectum and colon and extends proximally in a continuous fashion. Upon presentation, lesions are limited to the rectum (proctitis) in $30-35 \%$ of patients, to the splenic flexure in $30-45 \%$ and to the cecum (pancolitis) in 20-25\%. Mucosal lesions are usually diffuse and superficial, and deep ulcerations are present only in patients with severe disease. Perianal disease may develop in rare cases of UC. Extraintestinal manifestations are observed in one-third of UC patients. In UC, the main complications include toxic megacolon, massive hemorrhage or colon perforation, while strictures and fistulas are uncommon [2, 3].

In $\mathrm{CD}$, the lesions can involve any segment of the digestive tract, from the mouth to the anus, but mainly affect the distal ileum and the colon. At the time of diagnosis, approximately $40 \%$ of patients present with ileocolonic disease, about $30 \%$ have isolated ileal disease, and another $30 \%$ have a pure colonic disease. Approximately $5-15 \%$ of patients have associated upper gastrointestinal lesions and $20-30 \%$ present perianal lesions. Other patients have a more severe course and may develop complications that require surgery. In CD, intestinal strictures, internal or perianal 
fistulas or abscesses are frequent, being reported in approximately one-third of patients $[2,3]$.

CD and UC are incurable; they begin in young adulthood and continue throughout life. Prognosis is difficult to determine. The mortality of patients with UC is not greater than that of the general population, but patients with CD have greater mortality than the general population. It has been proposed that only aggressive therapeutic approaches, based on treatment of early recurrent lesions in asymptomatic individuals, have a significant impact on progression of these chronic diseases. The overall aging of the population suggests that the burden of disease due to IBD in the elderly will continue to increase. Recent studies have identified an increasing incidence of hospitalizations related to IBD. Older patients with IBD-related hospitalizations have higher mortality than younger patients and higher hospitalization charges $[1,4]$.

It is difficult to establish the true incidence of IBD in older patients due to problems of case definition, population, and particularly because it may be confused with other clinical conditions. Old age colitis refers to patients $>60$ years of age who are affected by a group of diseases of the colon, including infections, vasculitis, microscopic colitis, colorectal cancer, ischemic colitis, drug-associated colitis (particularly non-steroidal anti-inflammatory drugs) and IBD [5]. Special precautions must be taken in the diagnosis of these patients to avoid errors, and new diagnostic tools might be useful for a correct diagnosis.

\section{Case Report}

A 91-year-old man was admitted to the Emergency Department with progressively worsening abdominal pain and 2-4 episodes of bloody diarrhea daily for the last month. Physical examination revealed normal temperature, pulses, blood pressure and oxygen saturation. Similar symptoms were not reported by the patient or his family during the past. Complete blood count and biochemical tests showed normal hemoglobin, white blood cells, platelets, ESR and CRP levels, while tumor markers were negative. Stool examination showed erythrocytes and white blood cells, while stool culture was negative for parasites, eggs, bacteria and Clostridium difficile.

Chest and upper abdominal CT scan was negative, while pelvic CT showed inflammatory changes and loss of homogeneity in the perirectal fat together with considerable bowel wall thickening of both the rectum and sigmoid. Colonoscopy revealed edema, hyperemia and spontaneous friability presenting as a continuous mucosal injury, diffuse granular mucosal pattern and microulcerations of the rectosigmoid mucosa (fig. 1). The endoscopic view was compatible with UC. Tissue biopsies revealed diffuse mucosal inflammation with basal plasmacytosis of the lamina propria, neutrophilic cryptitis, crypt abscesses and mucosal crypt distortion, overall histopathological lesions compatible with UC (fig, 2).

The patient was treated with the antibiotics metronidazole and ciprofloxacin intravenously, as well as mesalazine, with clear clinical improvement during the 5th day of treatment, and was finally discharged with almost normal stools. After a 6-month follow-up, no recurrence has occurred.

\section{Discussion}

Herein, we report a case of first diagnosis of IBD in a 91-year-old man. There are minor data in the literature regarding IBD in patients $>85$ years of age. The aging of the population, especially in Western societies, might have an impact on the occurrence of certain diseases in very old ages. In our case, endoscopy and imaging raised the 
suspicion of IBD, while histological examination was diagnostic. On the contrary, blood tests were not helpful for diagnosis, as white blood cells were normal and inflammatory markers were negative. This might be explained by the fact that the main stimulator of CRP synthesis in the liver is interleukin 6 (IL-6), and studies have found a delayed IL-6 response in infection in the old as well as impaired production of proinflammatory cytokines. Thus, a diminished or delayed inflammatory response to infections in the old may be biologically plausible [6].

The clinical manifestations of the first flare of disease, both in UC and CD, are similar in the group aged $>60$ years and younger age groups. It has been noted that the severity of the symptoms of UC, particularly rectal bleeding and diarrhea, may be lower in patients in this group, but it may also have atypical forms of presentation, such as constipation [7]. With regard to disease location, according to the Montreal classification, proctitis and left-sided UC are more common in patients $>60$ years old than in younger patients. In the case of $\mathrm{CD}$, it has been observed that the form of disease presentation is similar between the different age groups, but that colonic disease location is the most common form in the older group [8]. With regard to disease behavior, the most common is inflammatory disease behavior, the stricturing and penetrating pattern being less common than in the 18-60 years age group [9].

It is often difficult to establish the diagnosis of IBD in older patients because the disease is uncommon and the initial symptoms are often mild or atypical. There are various clinical situations that may hinder diagnosis of IBD in elderly patients. IBD may be complicated by infections or by certain medications, such as non-steroidal anti-inflammatory drugs and antibiotics. Similarly, certain neoplasms, and specifically small bowel lymphoma, may have similar characteristics to those of IBD or be a complication of the disease itself $[5,10]$. Of particular relevance is segmental colitis associated with diverticular disease (SCAD), which may simulate IBD both clinically, endoscopically and histologically $[11,12]$. Diverticulosis affects more than half of the people $>60$ years of age. SCAD is clinically manifested by abdominal pain, rectal bleeding and altered bowel habits, but only $3-8 \%$ of patients have mucosal inflammation around the diverticula. A recent study in a German IBD elderly population showed that $8 \%$ of older patients diagnosed as IBD patients were in fact affected by SCAD [13].

The location, extent, severity and disease behavior, both in UC and CD, will determine the medical treatment options to be used. The principles governing medical management of IBD in patients aged $>60$ years are the same as in other age groups [5]. Oral and/or topical mesalazine is the main option for medical treatment in patients with a mild to moderate flare of UC. However, in CD, the efficacy of mesalazine is questionable [14]. Beclomethasone combined with mesalazine has been shown to be effective for treating moderate flares of left UC when administered orally as a single dose [15]. The prevalence of corticosteroid resistance or dependence in this group of patients has not been specifically evaluated, but it is estimated at about $30 \%$ [16]. There is no evidence of differences in terms of efficacy, metabolism and toxicity between patients aged $>60$ years and younger patients for conventional immunomodulatory agents such as azathioprine, mercaptopurine and methotrexate [14]. The indications of biological agents, such as infliximab, remain similar to those for patients aged $<60$ years. However, one of the most important aspects regarding the use 
of these agents is the safety profile, particularly that related to the risk of infections [17].

In general, the need for surgery is lower in CD patients with an age at diagnosis $>40$ years and colonic disease location [18]. In UC, restorative proctocolectomy with ileal pouch-anal anastomosis continues to be the surgical technique of choice. In general, results are not as good in older patients compared to younger patients, but they are both reasonably good in terms of efficacy and morbidity [19]. Finally, there were more hospitalizations in patients $>65$ years than in the younger group, representing $25 \%$ of all admissions for IBD occurring in the USA. In general, the disease course of UC is more severe in elderly patients and mortality in hospitalized patients, both with UC and CD, is estimated to be 3-5 times higher than in age subgroups $<65$ years [4]. In UC, age at diagnosis is not associated with increased mortality, whereas in CD, there is a slight increase in the risk of mortality in older patients ( $>55$ years) with long-standing disease [20].

In conclusion, we reported a case of first diagnosis of IBD in a 91-year-old man. The prevalence of IBD in patients aged $>80$ years is difficult to determine. Diagnostic tools are the same as for other age groups, but diagnosis may be difficult because there are a number of clinical conditions that may mimic IBD at this age. The treatment options are those used in younger patients, but special precautions should be taken.

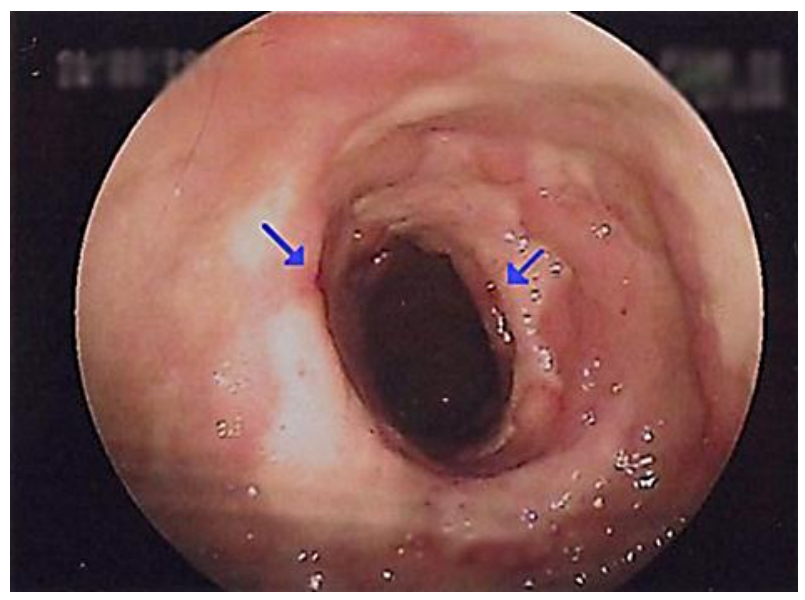

Fig. 1. Colonoscopy revealed edema, hyperemia and spontaneous friability presenting as a continuous mucosal injury and microulcerations (arrows) of the rectosigmoid mucosa. 


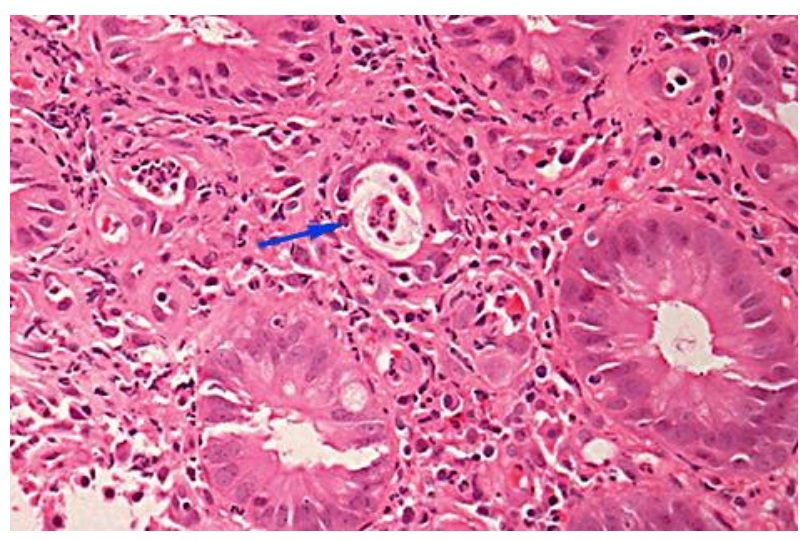

Fig. 2. Tissue biopsies revealed diffuse mucosal inflammation, cryptitis, crypt abscesses (arrow) and mucosal crypt distortion, overall histopathological lesions compatible with UC.

\section{References}

1 Cosnes J, Gower-Rousseau C, Seksik P, Cortot A: Epidemiology and natural history of inflammatory bowel diseases. Gastroenterology 2011;140:1785-1794.

$>2$ Vatn MH: Natural history and complications of IBD. Curr Gastroenterol Rep 2009;11:481-487.

-3 Latella G, Papi C: Crucial steps in the natural history of inflammatory bowel disease. World J Gastroenterol 2012;18:3790-3799.

-4 Ananthakrishnan AN, McGinley EL, Binion DG: Inflammatory bowel disease in the elderly is associated with worse outcomes: a national study of hospitalizations. Inflamm Bowel Dis 2009;15:182-189.

5 del Val JH: Old-age inflammatory bowel disease onset: a different problem? World J Gastroenterol 2011;17:2734-2739.

6 Wester AL, Blaasaas KG, Wyller TB: Is the concentration of C-reactive protein in bacteraemia associated with age? Immun Ageing 2008;5:8.

7 Grimm IS, Friedman LS: Inflammatory bowel disease in the elderly. Gastroenterol Clin North Am 1990;19:361-389.

8 Wagtmans MJ, Verspaget HW, Lamers CB, van Hogezand RA: Crohn's disease in the elderly: a comparison with young adults. J Clin Gastroenterol 1998;27:129-133.

\$9 Heresbach D, Alexandre JL, Bretagne JF, Cruchant E, Dabadie A, Dartois-Hoguin M, Girardot PM, Jouanolle H, Kerneis J, Le Verger JC, Louvain V, Pennognon L, Richecoeur M, Politis J, Robaszkiewicz M, Seyrig JA, Tron I: Crohn's disease in the over-60 age group: a population based study. Eur J Gastroenterol Hepatol 2004;16:657-664.

10 Brandt LJ: Bloody diarrhea in an elderly patient. Gastroenterology 2005;128:157-163.

11 Picco MF, Cangemi JR: Inflammatory bowel disease in the elderly. Gastroenterol Clin North Am 2009;38: 447-462.

12 Harpaz N, Sachar DB: Segmental colitis associated with diverticular disease and other IBD look-alikes. J Clin Gastroenterol 2006;40(suppl 3):S132-S135.

13 Hadithi M, Cazemier M, Meijer GA, Bloemena E, Felt-Bersma RJ, Mulder CJ, Meuwissen SG, Pena AS, van Bodegraven AA: Retrospective analysis of old-age colitis in the Dutch inflammatory bowel disease population. World J Gastroenterol 2008;14:3183-3187.

14 Van Assche G, Dignass A, Reinisch W, van der Woude CJ, Sturm A, De Vos M, Guslandi M, Oldenburg B, Dotan I, Marteau P, Ardizzone A, Baumgart DC, D’Haens G, Gionchetti P, Portela F, Vucelic B, Soderholm J, Escher J, Koletzko S, Kolho KL, Lukas M, Mottet C, Tilg H, Vermeire S, Carbonnel F, Cole A, Novacek G, Reinshagen M, Tsianos E, Herrlinger K, Bouhnik Y, Kiesslich R, Stange E, Travis S, Lindsay J: The second European evidence-based consensus on the diagnosis and management of Crohn's disease: special situations. J Crohns Colitis 2010;4:63-101. 
15 Manguso F, Balzano A: Meta-analysis: The efficacy of rectal beclomethasone dipropionate versus 5-aminosalicylic acid in mild to moderate distal ulcerative colitis. Aliment Pharmacol Ther 2007;26: 21-29.

16 Akerkar GA, Peppercorn MA, Hamel MB, Parker RA: Corticosteroid-associated complications in elderly Crohn's disease patients. Am J Gastroenterol 1997;92:461-464.

17 Ljung T, Karlen P, Schmidt D, Hellstrom PM, Lapidus A, Janczewska I, Sjoqvist U, Lofberg R: Infliximab in inflammatory bowel disease: clinical outcome in a population based cohort from Stockholm County. Gut 2004;53:849-853.

18 Tremaine WJ, Timmons LJ, Loftus EV Jr, Pardi DS, Sandborn WJ, Harmsen WS, Thapa P, Zinsmeister AR: Age at onset of inflammatory bowel disease and the risk of surgery for non-neoplastic bowel disease. Aliment Pharmacol Ther 2007;25:1435-1441.

19 Delaney CP, Fazio VW, Remzi FH, Hammel J, Church JM, Hull TL, Senagore AJ, Strong SA, Lavery IC: Prospective, age-related analysis of surgical results, functional outcome, and quality of life after ileal pouch-anal anastomosis. Ann Surg 2003;238:221-228.

-20 Wolters FL, Russel MG, Sijbrandij J, Schouten LJ, Odes S, Riis L, Munkholm P, Bodini P, O’Morain C, Mouzas IA, Tsianos E, Vermeire S, Monteiro E, Limonard C, Vatn M, Fornaciari G, Pereira S, Moum B, Stockbrugger RW: Crohn's disease: increased mortality 10 years after diagnosis in a Europe-wide population based cohort. Gut 2006;55:510-518. 